\title{
Adaptive Sliding Mode Control for a Constant Tension Suspension System
}

\author{
Yuxin Jia \\ The Seventh Research Division and the Center for Information and Control, School of Automation Science and \\ Electrical Engineering, Beihang University (BUAA), \\ Beijing, 100191, China \\ Yingmin Jia* \\ The Seventh Research Division and the Center for Information and Control, School of Automation Science and \\ Electrical Engineering, Beihang University (BUAA), \\ Beijing, 100191, China \\ Kai Gong \\ The Seventh Research Division and the Center for Information and Control, School of Automation Science and \\ Electrical Engineering, Beihang University (BUAA), \\ Beijing, 100191, China \\ E-mail:yuxinjia@buaa.edu.cn,ymjia@buaa.edu.cn,buaa_gk@buaa.edu.cn \\ www.buaa.edu.cn
}

\begin{abstract}
The constant tension suspension system (CTSS) can be used to counteract the gravity of experimental spacecraft, and is widely used in the ground test of spacecraft control schemes. In this paper, the mechanical model of a CTSS is established, and an adaptive sliding mode controller (ASMC) is designed for the CTSS. The simulation results show that the transient time and steady-state error of the ASMC system are small, which satisfies the requirements of microgravity simulation.
\end{abstract}

Keywords: constant tension suspension system, microgravity simulation, sliding mode control, adaptive law

\section{Introduction}

Space technology is a competitive focus of the cuttingedge technology in the world today. Many countries have successively carried out a series of major projects, such as space station construction, deep space exploration, and satellite navigation. These achievements indicate that mankind has entered a new stage of space exploration. On the other hand, higher requirements are also put forward for the reliability of the spacecraft control scheme. ${ }^{1-4}$ Because spacecraft work in an area which is difficult for humans to reach, space technology is the research field that relies on ground experiments. The ground test of spacecraft control scheme can not only reduce the risk of actual operation in space, but also shorten the development cycle. Therefore, how to establish microgravity environment on the ground to simulate the real motion of spacecraft is a major demand. ${ }^{5}$ Many research institutions have developed different kinds of devices to simulate the microgravity environment. The methods mainly include free fall, parabolic flight, suspension, liquid and air floatation. The

${ }^{*}$ Corresponding author.

(C) The 2021 International Conference on Artificial Life and Robotics (ICAROB2021), January 21 to 24, 2021 
suspension method balances the gravity of experimental spacecraft by adjusting the tension of hanging wire. The system has a simple structure and low cost. It not only overcomes the disturbance of air and liquid floatation on the motion of experimental spacecraft, but also can be used for long-time microgravity simulation. ${ }^{6}$ Japan, the United States, Canada, China and other countries have applied the suspension method to microgravity simulation for the ground experiments of spacecraft. In 2019, China Academy of Space Technology (CAST) used the suspension method to counteract part of the gravity of the Mars probe, verifying the performance of the probe control scheme in the Martian gravity environment. The system consists of a 140-meter-high test tower, a followup system and a Mars surface simulation area, which satisfies the requirements of the Mars environment simulation experiment. The constant tension suspension system (CTSS) is the key component of suspended microgravity experimental device. It is of great significance to research how to guarantee that the output force of CTSS is constant under disturbance.

An adaptive sliding mode controller (ASMC) is proposed for the CTSS in this paper. Compared with the control method in Ref. 5, the stability of the system is improved.

\section{System Modeling}

The structure of the CTSS studied in this paper is shown in Fig. 1, including a micro delay servo module, a low stiffness structure, an unconstrained structure, a universal joint, a lifting unit, and an experimental spacecraft. ${ }^{5}$

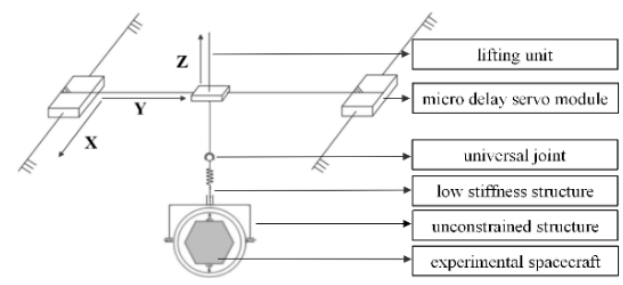

Fig. 1. Structure of the CTSS

The micro delay servo module is composed of $\mathrm{X}$ direction unit and Y-direction unit. It can measure the inclination of low stiffness structure by tilt sensor, and drive the lifting unit to track the movement of experimental spacecraft quickly, reducing the disturbance of the horizontal component of output tension on the movement of experimental spacecraft. The universal joint and unconstrained structure can ensure that the freedom of experimental spacecraft is not restricted, so that the experimental spacecraft can perform approximate unconstrained pitching, yawing and rolling motion under a small frictional torque disturbance. The vertical movement of lifting unit ensures that the output tension of the CTSS is always equal to the gravity of experimenttal spacecraft, realizing the long-term simulation of microgravity environment. The physical meanings of the main symbols are as follows:

- $g$, Gravitational acceleration.

- $R$, Radius of the gear in lifting unit.

- $k$, Elastic coefficient of low stiffness structure.

- $\omega$, Swing angle between lifting unit and low stiffness structure.

- $L_{0}$, Initial length of low stiffness structure.

- $L$, Variation length of low stiffness structure.

- $M$, The sum of the masses of unconstrained structure and experimental spacecraft.

- $T_{\alpha}$, Driving torque of the motor in lifting unit.

- $M_{1}, M_{2}$, The loads of the motors in X-direction and Ydirection units.

- $M_{a}, M_{b}$, The masses of the gear and rack in lifting unit.

- $F_{x}, F_{y}$, Driving forces of the motors in X-direction and Y-direction units.

- $\delta_{x}, \delta_{y}, \delta_{z}$, Disturbing forces

- $\omega_{x}, \omega_{y}$, Orthogonal decomposition values of $\omega$. According to the operation principle of CTSS, the model can be obtained by mechanical analysis.

$$
\boldsymbol{H} \ddot{\boldsymbol{q}}+\boldsymbol{J} \dot{\boldsymbol{q}}+\boldsymbol{N}=\boldsymbol{F}+\tilde{\boldsymbol{H}} \boldsymbol{\delta}
$$

Where $\boldsymbol{F}$ is the control vector of the CTSS, $\boldsymbol{q}$ is the state vector. $\delta$ is the external disturbance, and it is assumed that $\delta$ is bounded. The matrices defined in the model is as follows:

$$
\begin{aligned}
\boldsymbol{H} & =\left[\begin{array}{ccc}
-\omega_{x}\left(\eta_{1}-1\right) & -d\left(\eta_{1}-1\right) & 0 \\
-\omega_{y}\left(\eta_{2}-1\right) & 0 & -d\left(\eta_{2}-1\right) \\
-R\left(M-M_{c}\right) / M & 0 & 0
\end{array}\right] \\
\tilde{\boldsymbol{H}} & =\left[\begin{array}{ccc}
0 & -\eta_{1} / d & 0 \\
0 & 0 & -\eta_{2} / d \\
R M_{\mathrm{c}} / M & -\omega_{x} R M_{\mathrm{c}} /(M d) & -\omega_{y} R M_{\mathrm{c}} /(M d)
\end{array}\right] \\
\boldsymbol{J} & =\left[\begin{array}{ccc}
-2 \dot{\omega}_{x}\left(\eta_{1}-1\right) & 0 & 0 \\
-2 \dot{\omega}_{y}\left(\eta_{2}-1\right) & 0 & 0 \\
0 & 0 & 0
\end{array}\right], \boldsymbol{F}=\frac{1}{M}\left[\begin{array}{l}
F_{x} \\
F_{y} \\
T_{\alpha}
\end{array}\right], \boldsymbol{\delta}=\frac{1}{M}\left[\begin{array}{l}
\delta_{z} \\
\delta_{x} \\
\delta_{y}
\end{array}\right] \\
\eta_{1} & =\left(M+M_{1}\right) / M, \eta_{2}=\left(M+M_{2}\right) / M
\end{aligned}
$$




$$
\begin{aligned}
& \boldsymbol{N}=\left[\begin{array}{c}
0 \\
0 \\
R\left(g+\left(M M_{b} g+L M_{c} k\right) / M^{2}\right)
\end{array}\right], \boldsymbol{q}=\left[\begin{array}{c}
L \\
\omega_{x} \\
\omega_{y}
\end{array}\right] \\
& d=L+L_{0}+M g / k, M_{c}=M_{a} / 2+M_{b}+M
\end{aligned}
$$

\section{ASMC Design}

In order to suppress the uncertain disturbance of experimental spacecraft during the operation of CTSS, a new ASMC is designed in this section. Define variables:

$$
\begin{gathered}
\boldsymbol{A}(\boldsymbol{q}, \dot{\boldsymbol{q}})=-\boldsymbol{H}^{-1}(\boldsymbol{J} \dot{\boldsymbol{q}}+\boldsymbol{N}) \\
\boldsymbol{B}(\boldsymbol{q})=\boldsymbol{H}^{-1}, \tilde{\boldsymbol{\delta}}=\boldsymbol{H}^{-1} \tilde{\boldsymbol{H}} \boldsymbol{\delta}=\left[\tilde{\boldsymbol{\delta}}_{1}, \tilde{\boldsymbol{\delta}}_{2}, \tilde{\boldsymbol{\delta}}_{3}\right]^{\mathrm{T}}
\end{gathered}
$$

The system model (1) is transformed into

$$
\ddot{\boldsymbol{q}}=\boldsymbol{A}(\boldsymbol{q}, \dot{\boldsymbol{q}})+\boldsymbol{B}(\boldsymbol{q}) \boldsymbol{F}+\tilde{\boldsymbol{\delta}}
$$

The simulation of total weightlessness environment is studied in this paper, so the expected value of $\boldsymbol{q}$ is defined as $\boldsymbol{q}_{d}=[0,0,0]^{\mathrm{T}}$. The system error is $\boldsymbol{e}=\boldsymbol{q}-\boldsymbol{q}_{\boldsymbol{d}}$, and the upper bound of $|\tilde{\boldsymbol{\delta}}|$ is $\boldsymbol{\beta}=\left[\beta_{1}, \beta_{2}, \beta_{3}\right]^{\mathrm{T}}$. Thus, Eq. (2) is transformed into

$$
\ddot{\boldsymbol{e}}=\boldsymbol{A}(\boldsymbol{q}, \dot{\boldsymbol{q}})+\boldsymbol{B}(\boldsymbol{q}) \boldsymbol{F}+\tilde{\boldsymbol{\delta}}-\ddot{\boldsymbol{q}}_{d}
$$

The switching function is defined as:

$$
\boldsymbol{s}=\boldsymbol{D e}+\boldsymbol{V} \operatorname{sgn}(\dot{\boldsymbol{e}})^{z}
$$

$\operatorname{sgn}(\dot{\boldsymbol{e}})^{z}=\left[\left|e_{1}\right|^{z} \operatorname{sign}\left(e_{1}\right),\left|e_{2}\right|^{z} \operatorname{sign}\left(e_{2}\right),\left|e_{3}\right|^{z} \operatorname{sign}\left(e_{3}\right)\right]^{\mathrm{T}}$

Where, $\boldsymbol{D}=\operatorname{diag}\left(d_{1}, d_{2}, d_{3}\right)$ and $\boldsymbol{V}=\operatorname{diag}\left(v_{1}, v_{2}, v_{3}\right)$ are positive definite matrices, $\boldsymbol{s}=\left[s_{1}, s_{2}, s_{3}\right]^{\mathrm{T}}, \boldsymbol{z}=\operatorname{diag}(z, z, z)$ and satisfies $1<z<2$.

Define $\varphi=\left[\varphi_{1}, \varphi_{2}, \varphi_{3}\right]^{\mathrm{T}}$ is the adaptive parameter of the ASMC, and the adaptive law is selected as:

$$
\dot{\boldsymbol{\varphi}}=\left[\frac{\left|s_{1}\right|}{f_{1}}, \frac{\left|s_{2}\right|}{f_{2}}, \frac{\left|s_{3}\right|}{f_{3}}\right]^{\mathrm{T}}
$$

Where, $f_{1}, f_{2}, f_{3}$ are all positive constants.

Assumption 1. The stable value $\hat{\boldsymbol{\varphi}}=\left[\hat{\varphi}_{1}, \hat{\varphi}_{2}, \hat{\varphi}_{3}\right]^{\mathrm{T}}$ of the adaptive parameter satisfies $\hat{\varphi}_{p}>\beta_{p}, p=1,2,3$.

Theorem 1. Under Assumption 1, the system error $\boldsymbol{e}$ will converge to $\mathbf{0}$, if the ASMC $\boldsymbol{F}$ is designed as:

$$
\begin{aligned}
& \boldsymbol{F}=\boldsymbol{F}_{e}+\boldsymbol{F}_{d}+\boldsymbol{F}_{s} \\
& \boldsymbol{F}_{e}=-\boldsymbol{B}(\boldsymbol{q})^{-1} \operatorname{diag}\left(\frac{1}{z v_{1}\left|\dot{\boldsymbol{e}}_{1}\right|^{z-1}}, \frac{1}{z v_{2}\left|\dot{\boldsymbol{e}}_{2}\right|^{z-1}}, \frac{1}{z v_{3}\left|\dot{\boldsymbol{e}}_{3}\right|^{z-1}}\right) \boldsymbol{D} \dot{\boldsymbol{e}} \\
& \boldsymbol{F}_{d}=\boldsymbol{B}(\boldsymbol{q})^{-1}\left(-\boldsymbol{A}(\boldsymbol{q}, \dot{\boldsymbol{q}})+\ddot{\boldsymbol{q}}_{\boldsymbol{d}}\right) \\
& \boldsymbol{F}_{s}=-\boldsymbol{B}(\boldsymbol{q})^{-1}(\operatorname{diag}(\boldsymbol{\varphi}) \operatorname{sign}(\boldsymbol{s}))
\end{aligned}
$$

When $\dot{\boldsymbol{e}}_{p}=0$, define

$$
1 /\left(z v_{p}\left|\dot{\boldsymbol{e}}_{p}\right|^{z-1}\right)=0, p=1,2,3
$$

Proof. The error of the adaptive parameter $\boldsymbol{\varphi}$ is defined as $\tilde{\boldsymbol{\varphi}}=\boldsymbol{\varphi}-\hat{\boldsymbol{\varphi}}$, and the positive definite scalar function is selected as:

$$
\begin{aligned}
& V_{L}=\frac{1}{2} s^{\mathrm{T}} s+\frac{1}{2} \tilde{\boldsymbol{\varphi}} \operatorname{diag}\left(f_{1}, f_{2}, f_{3}\right) \operatorname{diag}\left(\theta_{1}, \theta_{2}, \theta_{3}\right) \tilde{\boldsymbol{\varphi}} \\
& \operatorname{diag}\left(\theta_{1}, \theta_{2}, \theta_{3}\right)=\boldsymbol{z} \boldsymbol{V} \operatorname{diag}\left(|\dot{\boldsymbol{e}}|^{z-1}\right)
\end{aligned}
$$

The differentiation of Eq. (4) and Eq. (7) is

$$
\begin{aligned}
& \dot{\boldsymbol{s}}=\boldsymbol{D} \dot{\boldsymbol{e}}+\boldsymbol{z} \boldsymbol{V} \operatorname{diag}\left(|\dot{\boldsymbol{e}}|^{z-1}\right) \ddot{\boldsymbol{e}} \\
& \dot{V}_{L}=s^{\mathrm{T}} \dot{\boldsymbol{s}}+\tilde{\boldsymbol{\varphi}}^{\mathrm{T}} \operatorname{diag}\left(f_{1}, f_{2}, f_{3}\right) \operatorname{diag}\left(\theta_{1}, \theta_{2}, \theta_{3}\right) \dot{\tilde{\boldsymbol{\varphi}}}
\end{aligned}
$$

Substitute the ASMC Eq. (6) into Eq. (8)

$$
\begin{aligned}
\dot{V}_{L}= & \boldsymbol{s}^{\mathrm{T}} \operatorname{diag}\left(\theta_{1}, \theta_{2}, \theta_{3}\right)(-\operatorname{diag}(\boldsymbol{\varphi}) \operatorname{sign}(\boldsymbol{s})+\tilde{\boldsymbol{\delta}}) \\
& +\tilde{\boldsymbol{\varphi}}^{\mathrm{T}} \operatorname{diag}\left(\theta_{1}, \theta_{2}, \theta_{3}\right)\left[\left|\boldsymbol{s}_{1}\right|,\left|\boldsymbol{s}_{2}\right|,\left|\boldsymbol{s}_{3}\right|\right]^{\mathrm{T}} \\
= & \theta_{1}\left(\tilde{\boldsymbol{\delta}}_{1} \boldsymbol{s}_{1}-\hat{\varphi}_{1}\left|\boldsymbol{s}_{1}\right|\right)+\theta_{2}\left(\tilde{\boldsymbol{\delta}}_{2} \boldsymbol{s}_{2}-\hat{\varphi}_{2}\left|\boldsymbol{s}_{2}\right|\right) \\
& +\theta_{3}\left(\tilde{\boldsymbol{\delta}}_{3} \boldsymbol{s}_{3}-\hat{\varphi}_{3}\left|\boldsymbol{s}_{3}\right|\right) \leq 0
\end{aligned}
$$

According to the analysis of Eq. (9), the controller Eq. (6) can make the system stable. Further, substituting the controller Eq. (6) into the system error model Eq. (3), it can be found that the solution $(\boldsymbol{e}, \dot{\boldsymbol{e}})$ of the system error model will not make $\dot{V}_{L} \equiv 0$ except for the origin $(\mathbf{0}, \mathbf{0})$. Therefore, the system error $\boldsymbol{e}$ will converge to $\mathbf{0}$. The proof is completed.

\section{Experimental Analysis}

In this section, the ASMC is applied to the CTSS for simulation experiments. The adaptive parameter of the ASMC is dynamically adjusted according to the value of Eq. (4), and finally converge to a constant. In order to 
reduce the oscillation caused by the $\operatorname{Sign}(s)$, Eq. (10) is used to replace it in the simulation.

$$
s /(|s|+0.05)
$$

The parameters of the CTSS model Eq. (1) are $\mathrm{R}=0.02 \mathrm{~m}$, $\mathrm{M}=15.5 \mathrm{~kg}, \mathrm{M}_{a}=0.52 \mathrm{~kg}, \mathrm{M}_{b}=4 \mathrm{~kg}, \mathrm{M}_{1}=16 \mathrm{~kg}, \mathrm{M}_{2}=41.5 \mathrm{~kg}$, $\mathrm{k}=690 \mathrm{~N} / \mathrm{m}, \mathrm{L}_{0}=0.45 \mathrm{~m}, \mathrm{~g}=10 \mathrm{~m} / \mathrm{s}^{2}$. The pulse disturbance shown in Fig. 2 is applied to the system.

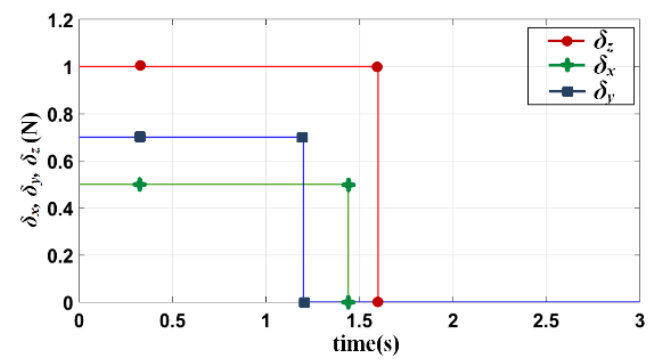

Fig. 2. External disturbance

The parameters of the ASMC are as follows:

$$
\begin{gathered}
f_{1}=f_{2}=f_{3}=1, \overline{\boldsymbol{\varphi}}=[1,1,1]^{\mathrm{T}}, z=1.1 \\
\boldsymbol{D}=\operatorname{diag}(280,510,450), \boldsymbol{V}=\operatorname{diag}(7.5,8,9)
\end{gathered}
$$

Where $\overline{\boldsymbol{\varphi}}$ is the initial value of the adaptive parameter.

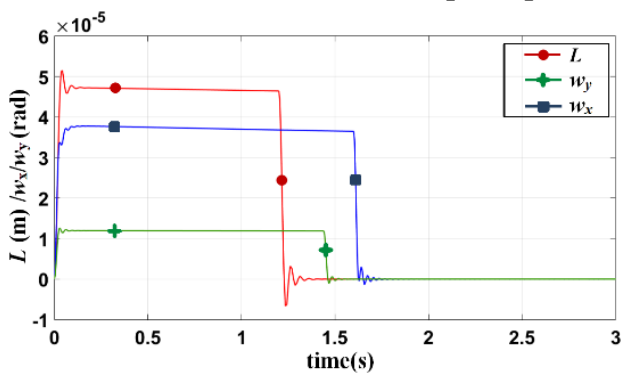

Fig. 3. State response of the ASMC system

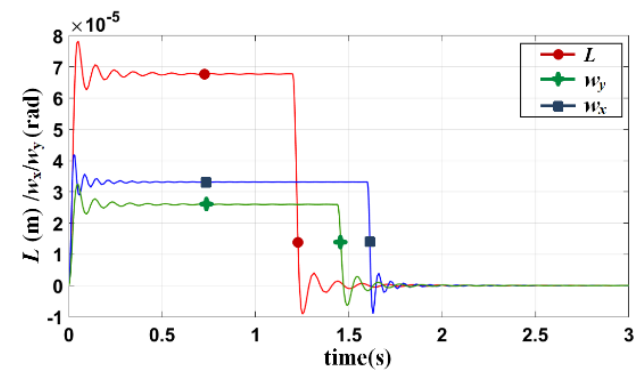

Fig. 4. State response of the SMC system in Ref. 5

The state response is shown in Fig. 3. It can be found that the maximum error of $\omega_{x}$ is $3.779 \times 10^{-5} \mathrm{rad}$, which is $0.0022^{\circ}$, the maximum error of $\omega_{y}$ is $1.254 \times 10^{-5} \mathrm{rad}$, which is $0.0007^{\circ}$, and the maximum error of $L$ is $5.151 \times 10^{-5} \mathrm{~m}$. Therefore, the output tension error of the system is $0.036 \mathrm{~N}$, which satisfies the requirements of microgravity simulation.

The SMC in Ref. 5 is also applied to the CTSS, and its state response is shown in Fig. 4. The results show that the convergence speed of the ASMC system is faster and the steady-state error is smaller. When the simulation time $\mathrm{t}>1.6 \mathrm{~s}$, the state error of the ASMC system satisfies $\|\boldsymbol{e}\|<4 \times 10^{-6}$, while the SMC system needs $1.7 \mathrm{~s}$.

\section{Conclusions}

A new ASMC is designed for the model of CTSS in this paper. The adaptive parameter can be dynamically adjusted with the value of the switching function. The asymptotic stability of the ASMC system is proved, and the simulation experiment is carried out to compare with the controller in Ref. 5. Experiments show that the ASMC system has smaller steady-state error and faster convergence speed, which satisfies the requirements of microgravity simulation.

\section{Acknowledgements}

This work was supported by the NSFC (61520106010, 61327807, 61134005, 61521091) and the National Basic Research Program of China (973 Program: 2012CB 821200, 2012CB821201).

\section{References}

1. Z. Jiang, J. Xu, H. Li, et al, Stable Parking Control of a Robot Astronaut in a Space Station Based on Human Dynamics, IEEE Trans. Robot., 36(2), 2020, pp. 399-413.

2. C. Wang, H. Nie, J. Chen, et al, The design and dynamic analysis of a lunar lander with semiactive control, Acta Astronaut., 157, 2019, pp. 145-156.

3. X. Liu, G. Cai, M. Wang, et al. Contact control for grasping a non-cooperative satellite by a space robot, Multibody Syst. Dyn., (5), 2020, pp. 119-141.

4. A. Witze, NASA rethinks approach to Mars exploration, Nature, 538(7624), 2016, pp. 149-150.

5. J. Jia, Y. Jia, S. Sun, Continuous Non-singular Fast Terminal Sliding Mode Control for an Active Gravity Field Simulator, Journal of Robotics Networking and Artificial Life, 3(4), 2017, pp. 253-256.

6. V. Arakelian, Gravity compensation in robotics, $A d v$. Robot., 30(2), 2016, pp. 79-96. 